\title{
The Long Run Effect of China's Higher Education Expansion on the Unemployment of Universities and Colleges Graduates
}

\author{
Mo Chen ${ }^{1,3}$ Wuquan Bei ${ }^{1}$ Yifan Wang ${ }^{1}$ Lin Zhao ${ }^{3}$ Jiaxin Sun ${ }^{3}$ Kyung Hyun Kim ${ }^{3, *}$
}

\author{
${ }^{1}$ Department of Education, Zhejiang Ocean University, Zhoushan, Zhejiang 316000, China \\ ${ }^{2}$ Zhoushan Museum, Zhoushan, Zhejiang 316000, China \\ ${ }^{3}$ Department of Education, Wonkwang University, Iksan-si 54538, Korea \\ *Corresponding author. Email: 395594829@qq.com
}

\begin{abstract}
Post-college unemployment has become a serious social problem in China. The current study draws data from the randomized samples from census data starting from 1999 to 2017. The relationship between research areas and labor market outcomes is examined. Universities have expanded their enrollment numbers, but the pathways to expansion and experienced structural changes vary greatly. We use nationally published data sets from 1999 to 2017 to investigate the medium and long run impacts that expansion policy has on unemployment of Universities graduates and College graduates (UGCG). The approach of Multivariate Time Series Analysis (MTSA) was employed to investigate the long term results associated with China's higher education expansion policy on UGCG.
\end{abstract}

Keywords: expansion unemployment rate, universities graduates and college graduates (UGCG),

Multivariate Time Series Analysis (MTSA)

\section{INTRODUCTION}

When listing the major indicators of any country's labor market, unemployment rate can't be omitted. Many researchers have come with different definitions of unemployment to evaluate labor market compactness along with demographic factors. However; it is a factor of an economy and high unemployment affect negatively. Very high unemployment rate in the economy may lead to weakening of purchase power parity due to low-income generation. It can also have negative influence on both local and international enterprises of the country if is not well controlled. Unemployment especially of the educated labor force in many cases is a waste of human capital which poses serious economic and social difficulties, and so it has attracted great interest to policy makers and researchers.

According to latest definition of youth population of World Health Organization, the estimated world youth population increased from 1.826 billion to 1.966 billion from 2018 to 2020 . The young people between 16 and 23 years old, who are suitable to studying in the universities and college, have occupied a big share

*Fund: MOE (Ministry of Education in China) Project of Humanities and Social Sciences (Project No.17YJC850016) : The comparative study on the villages' folk culture of islands between Chinese and Japanese. about $13.1 \%$ among of the world [1]. Meanwhile, the high number of unemployed youth is observed in in both developed and developing countries where it is often predominant among the University-educated workforce. Herein, we examine young universities and college graduates unemployment in China, with emphasis on the victims of higher education expansion.

China's education system is among the top ranking state-run education system world-wide. Since 1999, Chinese government over-enlarged the universities and colleges enrollment that has triggered many individual and social problems, making students and their family, government and society into dilemma. The wise action made by the government of China on enlarging the scale of higher education turned out well since the percentage of freshmen accepted to University went high by over $40 \%$ between year 1999 to 2000 . The rapid increase of new University students subsequently continued from 1999 to 2007 in a multiple means [2]. This big step ahead in higher education potentially opened more opportunity of higher education for youths. It seems to be a simple question to be answered, but is frequently, even so, raised in public argumentation. Particularly, the incrimination about the expansion policy for higher education is on the mouths of many people due to the higher number of unemployed ones, while others reasons that the rate of unemployed graduates would keep on being high. 
Others think this problem of unemployment is not only the outcome of expansion policy but also shows an image of low ability or a wage related matters among the graduates. Unluckily, there is insufficient empirical research speaking about these issues. Some contradictions upon the policy come up, and the points of contradictions includes the decline of quality of education, expense tuition fees, and growing in number of unemployed new Universities graduates and College graduates (UGCG).

Li, Whalley, and Xing (2014) in their casual observation and rigorous empirical analysis they argued that the policy of higher education expansion raised the figure of new unemployed universities and college graduates by $6 \%$ to $9 \%$ [3]. But above research was obviously lack of mega sample. That paper uses the periodic series of data from national census, for the period of 19 years from 1999 to 2018 to evaluate the impacts of expansion policy on unemployment for those with age range between 22-24. They realized there is a short-term effect, that is, only for those who have just come in the labor market. Will the consequences of unemployment persist for long-time? Do there have any changing criterions of unemployment for the affected cohorts? Variations in response to the posed questions have something to do with unstable labor market consequences brought upon expansion policy and thus have major policy necessary accompaniment. We came up with these queries basing more on new data from the 1999-2017 censuses. This particular information gives us access to trail the employment characteristics of the cohorts on observation for an extendible time period. Particularly, by equating the rate of unemployment for the identical cohort struck by the policy in 1999 (aged 22-24) as well as 2007 (aged 27-29), we observed that the rate of unemployment was at a low level for the duration of eight-years. While, the effect of expansion policy on unemployment rate in 2008 (aged 22-24) and 2017 (aged 27-29) was sharply increasing.

In order to investigate the effects of expansion policy on unemployment of the targeted cohorts in the long period of time, the strategies with Multivariate Time Series Analysis (MTSA) approaches has been used. The extension of assignation places of the UGCG is also a crucial factor to pay attention to in policy making and has potential influence on the results both at national level to local labor markets. Turned out that to certain extent the education level in urban areas compared to rural areas is high, several University graduates have their jobs and lives in cities. The year 2007 data's shows that University educated workers constitute about $15 \%$ of the labor force in China urban areas, and only $1 \%$ in the rural areas (Xing, 2016). Much more, within urban areas itself the University educated workers are unequally distributed. This has been due to cash flow where in the coastal areas tends to have higher income rate which corresponds to their education skills compared to small and med-sized cities hence more educated workers in bigger cities (Xing, 2016). There by, large cities are becoming more crowded and overwhelmed educated and larger. It's has always been the government wish for the University graduates to seek for job in small cities also in rural areas.

Xing (2016) discovered that the expansion policy of higher education had a short term impacts in central and northeastern regions compared to eastern and western regions, that means the number of unemployed graduates goes down quick in eastern and central but sluggish in western and northeastern regions. As more graduate workers from the west opt to be employed in coastal regions, our conclusive findings propose that the nature of labor market in coastal is not rigid in a sense that it take over the challenge of unemployment within a relatively short time period. [4]

This paper begins to give a way to long serial time analysis of higher education expansion policy in China including to the phase of three years colleges. Freeman (2009) and Li, Whalley, Zhang, and Zhao (2011) carried out about two early works that shows the major changes of higher education expansion policy in China. [2] Both two works pointed out employment tightness for University graduates as the results of expansion, but didn't include junior college students in the third grade and therefore do not fully cover the enrollment rate of China's college entrance examination.

The great worry have been arising that China's labor force is excessively educated with proportional to its industrial composition as the number of number of fresh University graduates goes higher while the rate of unemployment is still threatening disaster in the labor market. If the situation of unemployment prevails among the graduates, there will be every reason to put an eye on the ex-ante University premium, which minimizes the return to University education. Our final findings propose that the high rate of unemployment for new graduates from University is improbably to significantly affect the University premium. Lastly, as we consider the expansion of higher education as an external supply shock those results to unemployment, this work contributes among the literatures that explore about the long run impacts of negative employment experiences.

The configuration of this work is as follows. Section 2 discussed the background of the study. Section 3 briefly describes how to deal with the original data and summary statistics. Section 3 proposes model and the data analysis methods, as well as the software that was used to analyze the data. Enrollment rate as Dependent Variable, economic growth (GDP growth) and unemployment as Independent Variable, as mediating 
variables and work force, family income, population growth as moderating variables was done. The next is to discuss data and the summary statistics so that to look into how the higher education expansion policy influenced the rate of employment of University graduates in the long run.

\section{BACKGROUND}

Regardless of the economic changes and opening up, Chinese economy has achieved significant development and lives of the have been greatly transformed to the better life. China's unemployment rate has also changed dramatically. With the growth of population and the popularization of nine-year compulsory education, China's higher education has also undergone tremendous changes. Similarly to our study there are two following facets: scale of higher education expansion and modifications in the mechanism for equalizing the number of University graduates with employers. Although the proportional of higher education ceaseless becoming large, the rate of increase before 1998 was substantially lower compared with that from 1999 and ahead. In year 1999, the government of China started the policy of higher education expansion: the Chinese government maximized the admissions quota of higher education by about 0.55 million for 1999 . The maximum annual increase of new students in university started to be observed from 1978 (48\%). In the following years, the proportion of higher education kept on growing: the total number of universities were added from 1022 to 2442 from year 1998 to 2012, The registration of student flied up from 1.08 million to 6.89 million, the University graduates number from 0.83 million to 6.25 million, and the University students number from 3.41 million to 23.91 million. Number of university graduates expected to exceed 8.4 million by 2020 . In spite the fact that the policy might be ascertained within the economic situation bases, the expansion was unhopeful for several families which have high school graduates.

China's economy has been growing steadily from 1999 to 2007, and its unemployment rate has been gradually decreasing. However, since 2008, China's economic growth has slowed down, and its unemployment rate has also risen. Especially in the 2008 because of the world economic crisis, a large number of enterprises closed down, China's overall unemployment rate rose rapidly. Thereafter unemployment rate was stabilized at a certain level.

In addition with expansion policy, the government restructured the planning system for distributing University graduates to job positions. The market pressure of demand and supply and interactional agreements between the graduates and their employers mainly defines the employment of graduates. The growing in figure of University graduates looking for job in large coastal cities and in private sectors. The great sudden influx into the labor market of graduates from University as a result of the expansion policy contributes to major supply daze to the labor market. $\mathrm{Li}$ et al. (2014) observed that the rate of unemployment of fresh University graduates has potentially grown as a result of higher education expansion policy. [2] The disaster of Unemployment seems to be critical to fresh University graduates as their number tends to go higher approximately to 7 million in recent years. In a certain degree, Unemployment consequences to new university graduates made the government of China to stop fur more of higher education expansion in 2012. The enrollment number from 1.08 million students in 1998

1.6 million in 1999, was increasing to 7.3583 million in 2017. It has sharply increased by more than 7 times in 19 years.

Several steps have been taken by the Chinese government to overcome the employment stress of University graduates. The measures taken include the encouragement of University graduates to look of jobs in western regions as well as in rural areas. Much more the government encouraged entrepreneurship with great favor of credit granting and tax deduction from the government.

After the policy about the enrollment expansion of the college entrance examination carried out in 1999 , the number of students has been smoothly increasing; especially it reaches up a peak in 2010, the number of candidates more than 10 million. We can find that before 2005, the number of applicants and enrollment rates were basically linearly correlated. But after 2008, the candidates and enrollment rate has changed greatly. There is a growing gap between the number of applicants and the number of enrolments.

As policy of chin's higher education expansion carried out, the results of rates of unemployment in eastern, western and central regions are totally different. It is shown that western of China, because the demand of the above labor force is far less than the supply, the number of laid-off people waiting for employment, plus the new number of people waiting for employment every year, the average annual unemployment rate of in the western region is $8 \%$, which is far beyond than in eastern region.

\section{DATA}

To evaluate the long term impacts of unemployment of the expansion policy. We employed the randomized samples from census data starting from 1999 to 2017. The National Bureau of Statistics (NBS) obtains all information by following the same process of random sampling, taking place over 31 provinces, municipalities, and autonomous regions. The numbers 
of every single person observed in these data are 1,180 , $110,2,585,481$, and 1,267, 381 before modification. Our evaluations primarily based on the observations only with four-year University and above education degrees of college students. Since many of University urban samples for our evaluations. For comparative purposes, we as well keep those with high school and professional (3year) University degrees. Though these observations maybe systematically varying from University graduates, they give potential information for us to determine the employment circumstances in different years.

As for the Chinese high school students at what's age to take the entrance examination, according to the statistics bureau general statistics and our sample estimate. We assume that one starts their primary school at age 6 and attempts the University entry exam at age 18. For the one who didn't go to University, the assumption is they would have attempted the exam at age 18. Therefore, every individual has an ideally proposed year for University entry examination. In the 3 datasets, the age restriction is between 22 to 35 . According to the year of study and age, we can find out who have and who haven't directly faced the impacts of the expansion policy. For instance, no observed impacts of expansion policy in the 1999 sample, those who have taken part in the University entry exam from 1983 to 1996, and. For the 2007 sample, the one aged between 22 to 24 were the victims of policy, while ages above 27 were not. For the 2017 sample, those affected by the policy in the 2007 sample are 5years older, so those aged between 27 and 29 were affected and those aged above 32 were not. graduates have their jobs in urban areas, we refer to the

As the samples are limited only to the one with high school and above degrees, the high school graduates' share lowered from $60.6 \%$ in 1999 to $50.6 \%$ in 2007 and so to $39.1 \%$ in 2017; the 3-year University graduates' share gradually raised from $25.9 \%$ in 1999 to $29.4 \%$ in 2007 and so to $32.5 \%$ in 2017 ; the 4-year University graduates share raised from $13.5 \%$ in 1999 to $20.0 \%$ in 2007 and so to $28.4 \%$ in 2017.As the result of higher education expansion so the number of young people going to universities has elevated.

\section{A. Model}

1) Hypothesis development for long term effect of unemployment rate on China's higher education expansion: With regard to relevant review above, some idea framework was developed to guide the study to achieve its objectives. The conceptual consists of independent, dependent, mediating variables and moderating variables. The research hypotheses was developed to show the association existing among the dependent variable, independent variables, mediating variables and moderating variables as shown in "Fig. 1": H1 shows how enrollment affects unemployment. H2 shows how GDP growth affect enrollment. H3 shows how gender mediates the effects of unemployment on economic growth. H4 shows how reign mediates the effects of enrollment on economic growth, H5 shows how work force moderates the effects of enrollment on economic growth, H6 shows how family income moderates the effects of enrollment on economic growth, and $\mathrm{H} 7$ shows how population growth moderates the effects of unemployment on economic growth

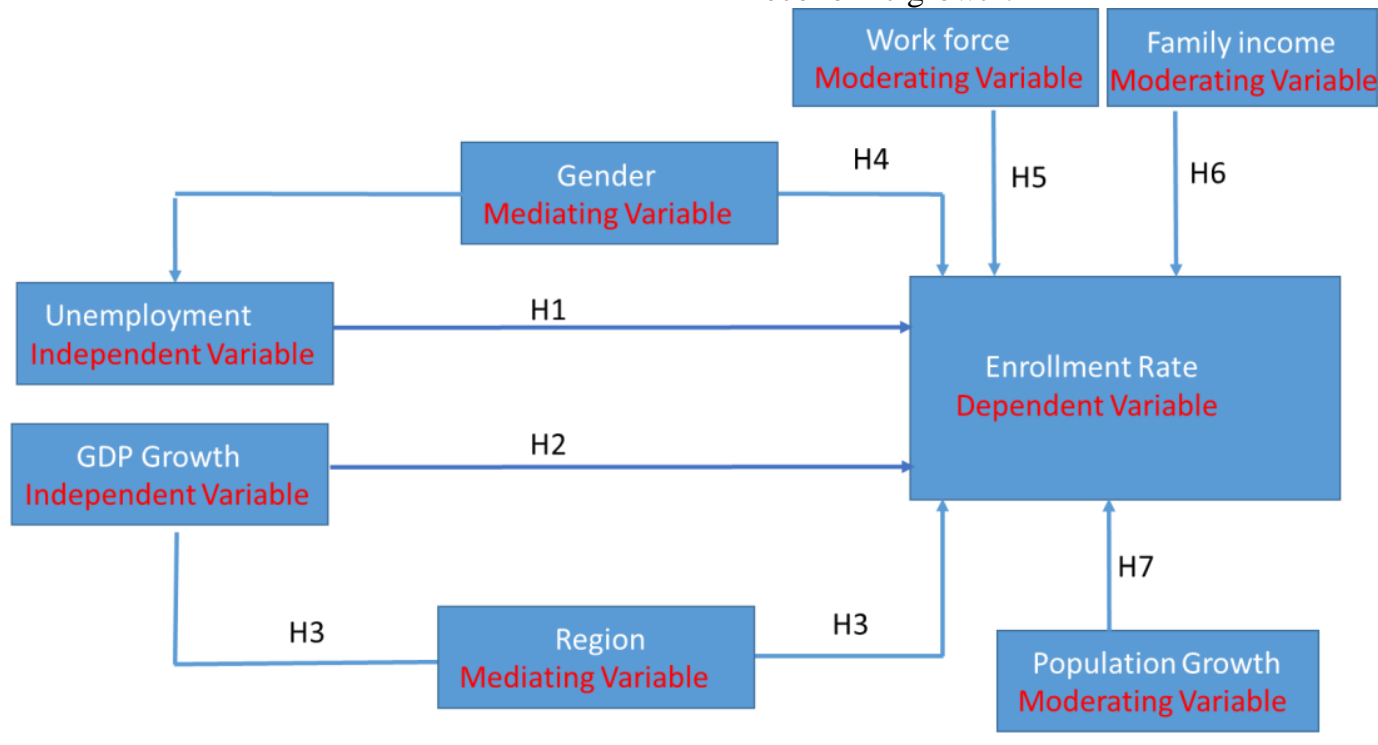

Fig. 1. Hypothesis development. 
2) Multivariate Time Series Analysis (MTSA): This study employed MTSA model similar to the one used by Fizari in his study; Multivariate Time Series Analysis on Correlation between Enrollment Rate and Unemployment Rate with Gross Domestic Product. Fizari's model used three main variables similar to this study. Therefore, this study applied the same model to examine the enrolment and unemployment affect China's economic growth from 1999 to 2017. Fizari's model was specified as:

$$
=\beta 0+\beta 1 X i+\beta 2 Z i+\mu t
$$

This model was expanded to add independent variables and moderating variables that were significant to the study as:

$\log ($ Enrollment Rate $)=\beta 0+\beta 1 \log ($ Unempl $)+\beta$ $2 \log (\mathrm{Wf})+\beta 3 \log (\mathrm{Fi})+$ ut $(1)$

$\log ($ Enrollment Rate $)=\beta 0+\beta 1 \log ($ GDP growth $)+$ $\beta 2 \log (\mathrm{PG})+\mathrm{ut}(2)$

Enrollment Rate =Admission ratio of Uinversity and Colleage Students (Dependent Variable) GDP growth $=$ Gross Domestic Product measure of Economic Growth (Independent Variable)

Unemr =Unemployment Rate (Independent Variable)

$\mathrm{Wf}=$ Work force $($ Moderation Variable)

$\mathrm{Fi}=$ Family Income (Moderation Variable)

$\mathrm{Pg}=$ Population Growth (Moderation Variable)

$\mathrm{UT}=$ Error Term

\section{$0,1,2,3$ are the estimation parameters}

This model was further modified to add mediating variables. The study employed a model similar to the one developed by Fizari's model. The general model for mediating variables was specified as:

$$
\begin{aligned}
& =i 1+c X+1 \\
& =\{2+c 1 X++2 \\
& =i 3+X+3
\end{aligned}
$$

$\mathrm{X}$ - Represents variable which are independent, Yrepresents variable which are dependent, and $\mathrm{M}$ - stands for the mediating variable. The edition model breaks down the total impacts of $\mathrm{X}$ on $\mathrm{Y}$ (c), into 2 parts: the indirect impact of $\mathrm{X}$ on $\mathrm{Y}$, quantified... Where $\mathrm{c}$ is the total effect of the independent variable on $Y$; $c^{\prime}$ is the impact of the independent variable on $\mathrm{Y}$ determining for $\mathrm{M}$; $\mathrm{b}$ is the results of the mediating factor on $\mathrm{Y}$; $\mathrm{a}$ is the outcome of the independent factor on the mediator;

í, $\mathbf{2} 2$, and $\mathbf{i} 3$ are the intercepts for each equation; and ut 1 , ut 2 , and ut 3 are the corresponding residuals in each equation. The expanded model to add mediating variables for the study was specified as follows:

$$
\begin{aligned}
& \log (\text { Enrollment Rate })=\beta 0+\beta 1 \log (\text { Unempl })+\beta \\
& 2 \log (\mathrm{Ge})+\text { ut }(3) \\
& (\text { Enrol })=1+2(\mathrm{Re})+ \\
& \log (\text { Enrollment Rate })=\beta 0+\beta 1 \log (\text { GDP growth })+ \\
& \beta 2 \log (\operatorname{Re})+\text { ut }(4) \\
& (\operatorname{Re})=1+2()+ \\
& \operatorname{Re}=\text { Region }(\text { i i i }) \\
& \text { Gen }=\text { Gender (i i i }) \\
& \text { Ut }=\text { Error Term }
\end{aligned}
$$

$0,1,2,3$ are the estimation parameters.

We employed random samples of the census information for 1999 , the $1 \%$ population study from 2001 to 2017 to evaluate the short and medium term impacts of expansion policy on employment. The National Bureau of Statistics (NBS) collects all data following similar procedures of random sampling, covering 31 provinces, municipalities, and autonomous regions. The numbers of individual observations in these data are $1,180,110,2,585,481$, and $1,267,381$ before cleaning. Our analysis mainly focuses on the observations with four-year University or above education degrees. As most University graduates work in urban areas, we consider the urban sample for our analysis. For comparison, we also keep those with high school degrees or professional (3year) University degrees. Although these observations might be systematically different from University graduates, they provide useful information for us to assess the employment situations in different years.

Because we do not have information on when people take the University entrance exam, we assume that one enters primary school at age 6 and takes the University entrance exam at age 18. For those who did not go to University, we assume they would have taken the exam at age 18. Thus, everyone has a hypothetical year for University entrance exam. In the three datasets, age is restricted to 22 to 35 . According to the year of survey and age, we can determine who are affected by the expansion policy and who are not. For example, those in the 1999 sample would have taken the University entrance exam between 1983 and 1996, and no observation was affected by the expansion policy. For the 2007 sample, those aged 22 to 24 were affected by the policy, and those aged above 27 were not. For 
the 2017 sample, those affected by the policy in the 2007 sample are 5years older, so those aged between 27 and 29 were affected and those aged above 32 were not.

A multiple regression method was used to analyze the association existing among the variables. The relationship of the independent, mediating, moderating and the dependent variables were established through the correlation and the regression equation.

3) Stationarity test: The estimate seems to be dynamic for a period of time due to the results of nonstationarity. This may result to unauthentic estimates. Non-stationary data can also lead to unreliable regression. Stationarity can be detected by the use of unit roots. In this research, stationarity test was done by dickey-fuller Test augmentation. The results showed that the variables were all stationary.

This research outlined the nature and the source of the data that was used for the study. The main source of the data was World Bank Database and covered 18 years (1999-2017). The model include enrollment as dependent variable, GDP growth and unemployment as independent variables, region and gender as the mediating variables, and work force, family income and population growth as the moderating variables. The data variables, the data analysis methods, discussion of the variables used as well.

\section{B. Data analysis and discussion}

Analyzing the time series data that was collected from various sources and has been summarized using summary statistics, these statistics also shows the tests that were done and includes the pairwise correlation test, variance enrollment factor test stationary test and linear regression test. The interpretation and discussion of the results as well as, the summary of results of hypotheses testing has also, been done in this section. The statistical summary of the data is given below in "Table I".

TABLE I. SUMMARY STATISTICS

\begin{tabular}{|c|c|c|c|c|c|}
\hline Variable & Obs. & Mean & Std. Dev & Min & Max \\
\hline Enrollment & 320 & 3.05 & 2.25 & -0.85 & 7.65 \\
\hline Economic growth (GDP growth) & 320 & 11.81 & 9.68 & 0.96 & 42.6 \\
\hline Unemployment & 320 & 9.05 & 0.25 & 9.5 & 12.3 \\
\hline Gender & 320 & 3.52 & 1.55 & 1.86 & 6.89 \\
\hline Region & 320 & 1.98 & 0.1 & 1.23 & 2.96 \\
\hline Work force & 320 & 8.57 & 8.39 & -0.82 & 21.5 \\
\hline Family income & 320 & 17.23 & 9.01 & 2.98 & 40.52 \\
\hline Population Growth & 320 & 2.76 & 0.25 & 2.65 & 3.35 \\
\hline
\end{tabular}

The data was collected from the years 1999 to 2017 . The dependent variable was economic growth measured by growth rate of GDP; two independent variables were enrollment and unemployment and the two mediating variables were Region and Gender. GDP growth had a standard deviation of 2.21557. The standard deviation of enrollment, unemployment, gender, region, family income, work force and population growth were 9.691389, 0.2819153, 1.581089, 0.4104246, 8.292514, 9.072374 , and 0.2447832 respectively. The minimum and the maximum values indicated in the table have helped in showing the range. The mean values were 3.087867 (GDP growth), 11.81229 (Enrollment), 9.704762 (Unemployment), 3.780571 (Region), 1.997975 (Gender), 8.587619 (Work Force), 17.15542 (Family Income) and 2.709777 (Population Growth).

\section{Discussion of the results}

This part outlines the discussion of the findings found from the data analysis. The section considered the effects of enrollment and unemployment on economic growth; mediation role of private investment and Gender; and the moderating role of interest rate, Family Income and population growth and their relationship on enrollment, unemployment and economic growth in China.

1) The effects of enrollment on economic development: Evaluation results showed that enrollment has a negative impacts on economic development of China (beta $=-0.6094705, \mathrm{P}=0.042$ ). The results confirm the previous studies by $\mathrm{Xu}$ (2016) that enrollment decreases the economic growth. However, the results contradict the study carried out by Li (2017) that higher rates of enrollment have both positive and negative 
effect on economic growth in Argentina, Bolivia, Brazil and Peru. The study partially supports because the effects of enrollment on economic growth vary from country to country, sector to sector and within subsectors based on income, research and development, culture, innovation activities and technological infrastructure. The government should use monetary and fiscal policies to control the enrollment level in the country. Gender can be used as an effective fiscal policy to maintain a desirable rate of enrollment. The government should reduce recurrent expenditure and other unnecessary expenditure on non-development activities in order to control enrollment. The authorities should put in place strict policies that will help in reducing the cost of production. It should also, minimize the importation responsibilities and maximize the exportation responsibilities in order, to increase the supply of goods and services in the country.

2) The effects of unemployment on economic development: The results of the analysis showed that unemployment has a negative impacts on economic development of China (beta $=-98.65854, \mathrm{P}=0.008$ ). The results confirm the study carried out by that unemployment has a unsupportive outcomes on economic development in Nigeria. This paper supports because China and Nigeria have similar characteristics in terms of technology, education, innovation activities, income and infrastructure. The government should invest more in education for the youths, in order to increase literacy rate and control the rate of unemployment. The government should also, fund young people with various ideas of innovation, entrepreneurship and encourage self-employment to reduce unemployment rate.

3) Mediating role of region: Investment on Unemployment and Economic Growth The results of the analysis showed that Region have a positive relationship with economic growth of China, but is not statistically significant (beta $=1.943109, \mathrm{P}=0.349$ ). The results confirm the study carried out by that Region has a supportive influence on economic growth in China, as well as mediating nemployment and economic growth. This study supports because China's private sectors contribute ignificantly to the economic growth. These sectors include infrastructure, research and development, technology and service industries. The government should create a good conducive environment for private sectors by providing good security, sufficient electricity and controlling enrollment in order to encourage more investments that lead to creation of more jobs and contribute to the economic growth. Region can also be used as an effective tool for the reduction of unemployment.
Expanded businesses create more job opportunities and increase the income level of citizens. Regions also encourage the consumption of goods and services in the economy, which contributes positively towards the national income.

4) Mediating role of gender on enrollment and economic growth: The results of the analysis showed that the association between Gender and economic development of China is positive (beta $=5.629512$, $\mathrm{P}=0.057)$. The results also, found that Gender is not statistically significant at $5 \%$ significant level. However, it is statistically significant at $1 \%$ significant level. The results confirm the study carried out that economic growth is positively affected by Gender on capital expenditure. However, the results contradict the study carried out that recurrent expenditure has a negative relationship with economic growth. This study supports because capital expenditure in China on sectors such as innovation activities, research and development, education and technological infrastructure have positive effect on economic growth. The government should increase their spending on various sectors such as infrastructure, industries, institutions for instance schools and medical institutions, as well as partner with private investors so that to boost the economic development. The government should minimize the recurrent expenditure and increase capital expenditure.

5) Moderating role of work force on enrollment and economic growth: The results of the analysis showed that Work Force moderates enrollment and economic growth in China (beta $=-0.7871822, \mathrm{P}=0.020$ ). The results confirm the study carried out that increase in interest rates affect economic growth negatively. The results contradict the study carried out that higher real rates of return facilitates greater savings, that as a result spur economic growth. The Central Bank of China should maintain interest rate at the desired level in order to control enrollment rate in the country. It should also provide interest free loans for youths to start up business would increase production of goods and services as well as consumption.

6) Moderating role of family income on enrollment and economic growth: The results of the analysis showed that Family Income moderates enrollment and economic growth in China (beta $=0.7545791, \mathrm{P}=0.013$ ). The results confirm the study conducted by that an increase in the Family Income in the economy lowers the interest rates, facilitating more borrowing/lending and consumption of the people thus contributing to rise in total output and GDP growth. The results contradict the study by that excess Family Income affect the enrollment rate in China in the short run. The Central 
Bank of China should ensure that the liquidity in the market is sufficient, maintain low interest rates in order to encourage savings and Regions. This measure would lead to increased income, more job opportunities and growth of the economy.

7) Moderating role of population growth on unemployment and economic growth: The results of the analysis showed that population growth moderates unemployment and economic growth in China (beta=83.69256, $\mathrm{P}=0.019$ ). The results confirm the study.conducted that population growth affect economic growth positively. However, the study contradict the study that population development have a unsupportive outcomes on economic development. The government should invest in the agricultural sector in order to produce enough food that will feed the growing population. This measure would as well curb food enrollment, increase job opportunities and stabilize the economy.

\section{CONCLUSION}

The worry on the unemployment of UGCG has been rising as China's higher education expanded. In this paper we have depicted that the higher education expansion in the long run impacts on high unemployment rate among University and college graduates. This finding indicates that the Chinese government should reduce the number of enrolment and increase the employment rate of students. Thus, existing studies provides a reliable evidence and information for government decision-making. We propose the encouragement of regional mobility of UGCG and the increment of matching quality that can prospectively trim down the unemployment rate national wide.

\section{References}

[1] S.M. Yasir Arafat, Bithika Mali, Hasina Akter. Is suicide reporting in Bangla online news portals sensible? A year-round content analysis against World Health Organization guidelines [J]. Asian Journal of Psychiatry, 2020, 49.

[2] Fanqin Meng, Xinjian Qu. A Survey of Degree Standards System in China's Higher Education $[\mathrm{P}]$. 2nd International Conference on Education, E-learning and Management Technology (EEMT 2017), 2017

[3] Zhou Jianping. On Career Guidance for University Graduates Who Haven't Get Employment [J] Journal of College Counselors, 2014, 6 (04): 70-74.

[4] Data Analytics Reports from Claremont Graduate University Advance Knowledge in Data Analytics (A snail shell process model for knowledge discovery via data analytics) [J]. Computers, Networks \& Communications, 2016.

[5] Lijun Yang. Higher education expansion and post-college unemployment: Understanding the roles of fields of study in China [J]. International Journal of Educational Development, 2018, 62 .
[6] Arria Amelia M, Garnier-Dykstra Laura M, Cook Emily T, Caldeira Kimberly M, Vincent Kathryn B, Baron Rebecca A O'Grady Kevin E. Drug use patterns in young adulthood and post-college employment. [J]. Drug and alcohol dependence, 2013, 127(1-3)

[7] JooHee Han. Who Goes to College, Military, Prison, or LongTerm Unemployment? Racialized School-to-Labor Market Transitions Among American Men [J]. Population Research and Policy Review, 2018, 37 (4). 